doi:10.13108/2020-12-2-97

\title{
REGULARITY OF ALMOST PERIODIC SOLUTIONS OF POISSON EQUATION
}

\author{
È. MUHAMADIEV, M. NAZAROV
}

\begin{abstract}
This paper discusses some regularity of almost periodic solutions of the Poisson equation $-\Delta u=f$ in $\mathbb{R}^{n}$, where $f$ is an almost periodic function. It wasproved by Sibuya [Almost periodic solutions of Poissonв $\hbar^{T M}$ S equation. Proc. Amer. Math. Soc., 28:195198, 1971.] that if $u$ is a bounded continuous function and solves the Poisson equation in the distribution sense, then $u$ is an almost periodic function. In this work, we weaken the assumption of the usual boundedness to boundedness in the sense of distribution, which we refer to as a bounded generalized function. The set of bounded generalized functions are wider than the set of usual bounded functions. Then, assuming that $u$ is a bounded generalized function and solves the Poisson equation in the distribution sense, we prove that this solution is bounded in the usual sense, continuous and almost periodic. Moreover, we show that the first partial derivatives of the solution $\partial u / \partial x_{i}, i=1, \ldots, n$, are also continuous, bounded and almost periodic functions. The technique is based on extending a representation formula using Green function for Poisson equation for solutions in the distribution sense. Some useful properties of distributions are also shown that can be used in studying other elliptic problems.
\end{abstract}

Keywords: Poisson equation, almost periodic solutions, generalized solutions.

Mathematics Subject Classification: 35J, 35D

\section{INTRODUCTION}

We study the following Poisson equation

$$
-\Delta u=f
$$

where $u: \mathbb{R}^{n} \rightarrow \mathbb{R}$ is the solution and $f: \mathbb{R}^{n} \rightarrow \mathbb{R}$ is a given almost periodic source function and $\Delta=\operatorname{div} \cdot \nabla$ is the Laplace operator. A function $f$ is called an almost periodic of $\boldsymbol{x}$ if $f$ is continuous in $\mathbb{R}^{n}$, and for each sequence of points $\left\{\boldsymbol{x}_{n}\right\} \in \mathbb{R}^{n}$, the corresponding sequence $\left\{f\left(\boldsymbol{x}+\boldsymbol{x}_{n}\right)\right\}$ contains a uniformly convergent subsequence. Our interest is to study the behavior of the solution, which is obtained when the source function is almost periodic.

The theory of almost periodic solutions of ordinary differential equations was started by early work of Bohr \& Neugebauer [1] and Jean Favard [3, 4]. Jean Favard proved the following theorem: if all homogeneous limit equations have no non-zero bounded solutions, and the original system has a bounded solution, then this solution is almost periodic. This result raised the problem of the existence of a bounded solution of a system with almost periodic coefficients. Later, it was shown by Muhamadiev [5], that the property of limit systems mentioned in the theorem of Favard guarantees the existence of a bounded solution of the original system, and consequently its almost periodicity.

The issue on the behavior of solutions to Poisson equation with almost periodic source functions was first addressed by Sibuya [7]. Later, Sell in [6] extended the result of Sibuya

E. Muhamadiev, M. Nazarov, Regularity of almost periodic solutions of Poisson equation.

(C) 2019 E. Muhamadiev, M. Nazarov.

This material is based upon work supported by Esseen scholarship at Uppsala University.

Submitted September 28, 2019. 
and Favard to linear systems of partial differential equations with almost periodic coefficients and source functions. We remark here that the extension of the theory of almost periodic solution to more general classes of differential equations such pseudo-differential operators was studied by Shubin [8].

The following theorem was proven by Sibuya [7].

Theorem 1.1 (Sibuya). Let $f(\boldsymbol{x})$ be an almost periodic function of $\boldsymbol{x}$ in $\mathbb{R}^{n}$, and let $u(\boldsymbol{x})$ be a bounded continuous function of $\boldsymbol{x}$ in $\mathbb{R}^{n}$. Assume that $u(\boldsymbol{x})$ is a solution of (1) in the sense of distribution. Then $u(\boldsymbol{x})$ is almost periodic with respect to $\boldsymbol{x}$ in $\mathbb{R}^{n}$.

Theorem 1.1 shows that if a bounded continuous function $u$ solves equation (1) in the distribution sense, then it is almost periodic. The main goal of this paper is to address the following key questions:

(i) to study the possibility of relaxing the assumption of Theorem 1.1, i.e., to consider a wider class of solutions rather than bounded continuous functions;

(ii) to study the properties of the partial derivatives of such solutions, i.e., boundedness, continuity, and almost periodicity.

We stress that in this paper we assume that the solution $u$ of the Poisson equation is bounded in the distribution sense, whereas in [7] and [6] the solution was assumed to be bounded in the strong sense.

This paper is organized as follows. Some definitions including that of the bounded generalized functions are presented in Section 1.1. We formulate our main results in Section 2. Main theorems are proved in Section 3. Some technical lemmas are proved in detail in Section 2.1 and in the Appendix.

1.1. Preliminaries. Throughout this work we follow the notations and definitions consistent with [2]. Let $\Omega$ be an open subset of $\mathbb{R}^{n}$, we denote its closure by $\bar{\Omega}$. We often use an open ball of radius $r>0$ centered at the point $\boldsymbol{x}$ denoted by $\mathcal{B}^{0}(\boldsymbol{x}, r)=\left\{\boldsymbol{y} \in \mathbb{R}^{n}:|\boldsymbol{x}-\boldsymbol{y}|<r\right\}$, and the symbol $\mathcal{B}(\boldsymbol{x}, r)=\left\{\boldsymbol{y} \in \mathbb{R}^{n}:|\boldsymbol{x}-\boldsymbol{y}| \leqslant r\right\}$ stands for a closed ball. For a given function $g(\boldsymbol{x}) \equiv g\left(x_{1}, \ldots, x_{n}\right), \boldsymbol{x} \in \Omega$, we denote the normal derivative by

$$
\frac{\partial g}{\partial \boldsymbol{\nu}}(\boldsymbol{x})=\boldsymbol{\nu} \cdot D g(\boldsymbol{x})
$$

where $\boldsymbol{\nu}$ is an outward pointing unit normal to the boundary $\partial \Omega$, and

$$
D g:=\left(\frac{\partial g}{\partial x_{1}}, \ldots, \frac{\partial g}{\partial x_{n}}\right)
$$

is the gradient of $g$. Furthermore, we use the so-called Green's functions to write the representation formula for the Poisson equations, see e.g., [2].

Definition 1. Green's function for an open set $\Omega=\mathcal{B}^{0}(\boldsymbol{x}, 1)$ in $\mathbb{R}^{n}$ is

$$
G(\boldsymbol{x}, \boldsymbol{y}):=\Phi(\boldsymbol{y}-\boldsymbol{x})-\Phi(|\boldsymbol{x}|(\boldsymbol{y}-\tilde{\boldsymbol{x}})), \quad(\boldsymbol{x}, \boldsymbol{y}) \in \Omega, \quad \boldsymbol{x} \neq \boldsymbol{y},
$$

where $\tilde{\boldsymbol{x}}=\frac{\boldsymbol{x}}{|\boldsymbol{x}|^{2}}$ and

$$
\Phi(\boldsymbol{x})= \begin{cases}-\frac{1}{2 n} \log (\boldsymbol{x}), & n=2 \\ \frac{1}{n(n-2) \alpha(n)} \frac{1}{|\boldsymbol{x}|^{n-2}}, & n \geqslant 3 .\end{cases}
$$

Theorem 1.2. (Representation formula) Let $\Omega=\mathcal{B}^{0}(\boldsymbol{x}, 1)$. If $u \in \mathcal{C}^{2}(\bar{\Omega})$ is the solution of (1), then the following identity holds

$$
u(\boldsymbol{x})=-\int_{\partial \Omega} u(\boldsymbol{y}) \frac{\partial G}{\partial \boldsymbol{\nu}}(\boldsymbol{x}, \boldsymbol{y}) \mathrm{d} S(\boldsymbol{y})+\int_{\Omega} f(\boldsymbol{y}) G(\boldsymbol{x}, \boldsymbol{y}) \mathrm{d} \boldsymbol{y} \quad(\boldsymbol{x} \in \Omega),
$$


where $\boldsymbol{\nu}$ is an outward normal vector, $\frac{\partial G}{\partial \boldsymbol{\nu}}(\boldsymbol{x}, \boldsymbol{y})$ is the normal derivative of function $G(\boldsymbol{x}, \boldsymbol{y})$ at point $\boldsymbol{y} \in \partial \Omega$.

We denote by $\mathcal{D}\left(\mathbb{R}^{n}\right)$ the set of all infinitely differentiable compactly supported functions $\phi: \mathbb{R}^{n} \rightarrow \mathbb{R}^{n}$ such that $\phi$. A function $u(\boldsymbol{x})$ is said to be a generalized or weak solution of (1) if it solves the following integral equation for every $\phi \in \mathcal{D}\left(\mathbb{R}^{n}\right)$ :

$$
-\int_{\mathbb{R}^{n}} u \Delta \phi \mathrm{d} \boldsymbol{x}=\int_{\mathbb{R}^{n}} f \phi \mathrm{d} \boldsymbol{x} .
$$

Next we give the definition of bounded generalized functions.

Definition 2 (Bounded generalized function). We say that the distribution $g(\boldsymbol{x})$ is a bounded generalized function in $\mathbb{R}^{n}$, if for any function $\varphi \in \mathcal{D}\left(\mathbb{R}^{n}\right)$, the function $(g * \varphi)(\boldsymbol{x})=(g(\boldsymbol{y}), \varphi(\boldsymbol{x}-\boldsymbol{y}))$ is bounded in $\mathbb{R}^{n}$, i.e., $\sup |(g * \varphi)(\boldsymbol{x})|<\infty$.

It can be easily observed that the set of bounded generalized functions contains the set of usual bounded functions.

\section{MAIN RESUltS}

In this section we formulate the main results of the paper. The first result extends the results by Sibuya [7]. We prove that under the same assumptions of Theorem 1.1, the function $u$ is continuously differentiable and its partial derivatives are almost periodic.

Theorem 2.1. Let $f(\boldsymbol{x})$ be an almost periodic function of $\boldsymbol{x}$ in $\mathbb{R}^{n}$, and let $u(\boldsymbol{x})$ be a bounded continuous function of $\boldsymbol{x}$ in $\mathbb{R}^{n}$. Assume that $u(\boldsymbol{x})$ is a solution of (1) in the sense of distribution. Then $u$ has continuous partial derivatives $\partial u / \partial x_{i}$, which are almost periodic functions of $\boldsymbol{x}$ in $\mathbb{R}^{n}$.

Theorem 2.1 generalizes the Sibuya's result in the case that not only $u$ is almost periodic, but also the partial derivatives $\partial u / \partial x_{i}$ are almost periodic. The second result of this work is to prove that $u$ does not have to be a bounded continuous function in the usual sense. We prove that if the weak solution of the Poisson equation is a bounded generalized function, then it is also a bounded continuous function in the usual sense.

Theorem 2.2. Let $u$ be a bounded generalized function in $\mathbb{R}^{n}$ which solves equation (1) in the distribution sense. Then $u$ is a continuous and bounded function in $\mathbb{R}^{n}$.

In Section 3.1 we prove Theorem 2.1, and Section 3.2 is devoted to the proof of Theorem 2.2. Before proving our main theorems, we need to prove several technical lemmata in the next section.

2.1. Technical lemmata. First, we prove that if $u$ is a solution of the Poisson equation in the distribution sense then it can be written via representation formula (4).

Lemma 2.1. Let $u(\boldsymbol{x})$ be a bounded continuous function of $\boldsymbol{x}$ in $\mathbb{R}^{n}$ that solves (1) in the sense of distribution. Then representation formula (4) holds for $u$.

Proof. We use the definition and properties of a standard mollifier. Let $\omega$ be a standard mollifier defined as

$$
\omega(\boldsymbol{x})= \begin{cases}c \exp \left(\frac{1}{|\boldsymbol{x}|^{2}-1}\right), & |\boldsymbol{x}|<1 \\ 0, & |\boldsymbol{x}| \geqslant 1\end{cases}
$$

such that

$$
\int_{|\boldsymbol{x}|<1} \omega(\boldsymbol{x}) \mathrm{d} \boldsymbol{x}=1
$$


Further, we define

$$
\omega_{\varepsilon}(\boldsymbol{x})=\frac{1}{\varepsilon^{n}} \omega\left(\frac{\boldsymbol{x}}{\varepsilon}\right),
$$

and let $u_{\varepsilon}:=\omega_{\varepsilon} * u$ and $f_{\varepsilon}:=\omega_{\varepsilon} * f$.

The proof consist of two steps. First, we show that the following identity holds:

$$
-\Delta u_{\varepsilon}(\boldsymbol{x})=f_{\varepsilon}(\boldsymbol{x}) \text {. }
$$

Then, we pass to the limit as $\varepsilon \rightarrow 0$, and use a uniform convergence property of the mollifier to get the desired result.

Using the definition of mollifier, we have

$$
\begin{aligned}
-\Delta u_{\varepsilon}(\boldsymbol{x}) & =-\int_{\mathbb{R}^{n}}\left(\Delta \omega_{\varepsilon}(\boldsymbol{x}-\boldsymbol{y})\right) u(\boldsymbol{y}) \mathrm{d} \boldsymbol{y}, \\
f_{\varepsilon}(\boldsymbol{x}) & =\int_{\mathbb{R}^{n}} \omega_{\varepsilon}(\boldsymbol{x}-\boldsymbol{y}) f(\boldsymbol{y}) \mathrm{d} \boldsymbol{y} .
\end{aligned}
$$

For a fixed $\boldsymbol{x}$ we set $\varphi(\boldsymbol{y})=\omega_{\varepsilon}(\boldsymbol{x}-\boldsymbol{y})$ we obtain

$$
-\int_{\mathbb{R}^{n}} \Delta \varphi(\boldsymbol{y}) u(\boldsymbol{y}) \mathrm{d} \boldsymbol{y}=\int_{\mathbb{R}^{n}} \varphi(\boldsymbol{y}) f(\boldsymbol{y}) \mathrm{d} \boldsymbol{y},
$$

which is in fact true, since $u$ solves the equation (1) in the distribution sense. Therefore, we conclude that (6) holds and since $u_{\varepsilon}$ is a smooth function the representation formula (4) can be written as

$$
u_{\varepsilon}(\boldsymbol{x})=-\int_{\partial \Omega} u_{\varepsilon}(\boldsymbol{y}) \frac{\partial G}{\partial \boldsymbol{\nu}}(\boldsymbol{x}, \boldsymbol{y}) \mathrm{d} S(\boldsymbol{y})+\int_{\Omega} f_{\varepsilon}(\boldsymbol{y}) G(\boldsymbol{x}, \boldsymbol{y}) \mathrm{d} \boldsymbol{y} .
$$

Next, we write the following relation

$$
\begin{aligned}
u_{\varepsilon}(\boldsymbol{x})-u(\boldsymbol{x}) & =\int_{\mathbb{R}^{n}} \omega_{\varepsilon}(\boldsymbol{x}-\boldsymbol{y}) u(\boldsymbol{y}) \mathrm{d} \boldsymbol{y}-u(\boldsymbol{x}) \\
& =\int_{\mathbb{R}^{n}} \frac{1}{\varepsilon^{n}} \omega\left(\frac{\boldsymbol{x}-\boldsymbol{y}}{\varepsilon}\right) u(\boldsymbol{y}) \mathrm{d} \boldsymbol{y}-u(\boldsymbol{x}) .
\end{aligned}
$$

Let us denote $-\boldsymbol{\xi}=\frac{\boldsymbol{x}-\boldsymbol{y}}{\varepsilon}$, then $\mathrm{d} \boldsymbol{y}=\varepsilon^{n} \mathrm{~d} \boldsymbol{\xi}$ and

$$
\begin{aligned}
u_{\varepsilon}(\boldsymbol{x})-u(\boldsymbol{x}) & =\int_{\mathbb{R}^{n}} \omega(\boldsymbol{\xi}) u(\boldsymbol{x}+\varepsilon \boldsymbol{\xi}) \mathrm{d} \boldsymbol{\xi}-u(\boldsymbol{x}) \int_{\mathbb{R}^{n}} \omega(\boldsymbol{\xi}) \mathrm{d} \boldsymbol{\xi} \\
& =\int_{\mathbb{R}^{n}} \omega(\boldsymbol{\xi})(u(\boldsymbol{x}+\varepsilon \boldsymbol{\xi})-u(\boldsymbol{x})) \mathrm{d} \boldsymbol{\xi} .
\end{aligned}
$$

Consequently, we get that

$$
\left|u_{\varepsilon}(\boldsymbol{x})-u(\boldsymbol{x})\right| \leqslant \sup _{\boldsymbol{x} \in \mathbb{R}^{n},|\boldsymbol{\xi}| \leqslant 1}|u(\boldsymbol{x}+\varepsilon \boldsymbol{\xi})-u(\boldsymbol{x})| .
$$

Hence, we conclude that if the right hand side goes to zero as $\varepsilon \rightarrow 0$, then $u_{\varepsilon} \rightarrow u$ uniformly and similarly, $f_{\varepsilon} \rightarrow f$. Now passing to the limit in (7) as $\varepsilon \rightarrow 0$, we obtain the desired result. The proof is complete.

We next prove that a weak solution of the Poisson equation in an open unit ball has continuous partial derivatives in this ball.

Lemma 2.2. Let a continuous in the unit ball $\mathcal{B}(0,1)=\{\boldsymbol{x}:|\boldsymbol{x}| \leqslant 1\}$ function $u(\boldsymbol{x})$ be a solution to equation (1) in the distribution sense in $\mathcal{B}^{0}(0,1)=\{\boldsymbol{x}:|\boldsymbol{x}|<1\}$. Then $u(\boldsymbol{x})$ is continuously differentiable for all $\boldsymbol{x} \in \mathcal{B}^{0}(0,1)$. 
Proof. Using the definition of solution (4) for $\boldsymbol{x} \in \mathcal{B}^{0}(0,1)$ and the definition of the Green's function (2), we get

$$
\begin{aligned}
\frac{\partial G}{\partial \boldsymbol{\nu}}(\boldsymbol{x}, \boldsymbol{y}) & =\sum_{i=1}^{n} y_{i} G_{y_{i}}(\boldsymbol{x}, \boldsymbol{y}) \\
& =-\frac{1}{n \alpha(n)} \frac{1}{|\boldsymbol{x}-\boldsymbol{y}|^{n}} \sum_{i=1}^{n} y_{i}\left(\left(y_{i}-x_{i}\right)-y_{i}|\boldsymbol{x}|^{2}+x_{i}\right) \\
& =-\frac{1}{n \alpha(n)} \frac{1-|\boldsymbol{x}|^{2}}{|\boldsymbol{x}-\boldsymbol{y}|^{n}} .
\end{aligned}
$$

This implies that the function $\frac{\partial G}{\partial \boldsymbol{\nu}}$ is continuously differentiable for $\boldsymbol{x} \in \mathcal{B}^{0}(0,1)$ and $\boldsymbol{y} \in \partial \mathcal{B}(0,1)$. Hence, the first term in the right hand side of (4) is a differentiable function for $\boldsymbol{x} \in \mathcal{B}^{0}(0,1)$.

Similarly, it follows from the definition of (2) that

$$
\begin{aligned}
\frac{\partial G}{\partial x_{i}} & =\frac{\partial \Phi}{\partial x_{i}}(\boldsymbol{y}-\boldsymbol{x})-\frac{\partial \Phi}{\partial x_{i}}(|\boldsymbol{x}|(\boldsymbol{y}-\tilde{\boldsymbol{x}})) \\
& =\frac{1}{n \alpha(n)}\left(\frac{y_{i}-x_{i}}{|\boldsymbol{y}-\boldsymbol{x}|^{n}}-\frac{y_{i}-x_{i}|\boldsymbol{y}|^{2}}{|| \boldsymbol{y}\left|\boldsymbol{x}-\frac{\boldsymbol{y}}{|\boldsymbol{y}|}\right|^{n}}\right) .
\end{aligned}
$$

The first term of the right hand side of $\frac{\partial G}{\partial x_{i}}$ has an integrable singularity when $\boldsymbol{y}=\boldsymbol{x}$. When $\boldsymbol{y} \neq 0$, the second term is continuous and bounded by

$$
\left|\frac{y_{i}-x_{i}|\boldsymbol{y}|^{2}}{|| \boldsymbol{y} \mid \boldsymbol{x}-\frac{\boldsymbol{y}}{|\boldsymbol{y}|^{n}}}\right| \leqslant \frac{2}{(1-|\boldsymbol{x}|)^{n}},
$$

which is integrable.

We conclude that the function $u(\boldsymbol{x})$ defined by the representation formula (4) in the unit ball $\Omega=\mathcal{B}^{0}(0,1)$

$$
u(\boldsymbol{x})=-\int_{\partial^{0}(0,1)} u(\boldsymbol{y}) \frac{\partial G}{\partial \boldsymbol{\nu}}(\boldsymbol{x}, \boldsymbol{y}) \mathrm{d} S(\boldsymbol{y})+\int_{\mathcal{B}^{0}(0,1)} f(\boldsymbol{y}) G(\boldsymbol{x}, \boldsymbol{y}) \mathrm{d} \boldsymbol{y} \quad\left(\boldsymbol{x} \in \mathcal{B}^{0}(0,1)\right),
$$

is continuously differentiable for $\boldsymbol{x} \in \mathcal{B}^{0}(0,1)$.

Remark 1. Note that Lemma 2.2 is also true as $f \in L^{\infty}(\mathcal{B}(0,1))$.

In the above lemma, we have proved that $u$ is differentiable if the Green's function and the normal derivative of the Green's function are differentiable with respect to $\boldsymbol{x}$. The following lemma completes Lemma 2.2 .

Lemma 2.3. Let a continuous in the unit ball $\mathcal{B}(0,1)=\{\boldsymbol{x}:|\boldsymbol{x}| \leqslant 1\}$ function $u(\boldsymbol{x})$ be a solution to equation (1) in the distribution sense in $\mathcal{B}^{0}(0,1)=\{\boldsymbol{x}:|\boldsymbol{x}|<1\}$. Then, the following limits hold:

$$
\begin{aligned}
& \lim _{h \rightarrow 0} \int_{\partial \mathcal{B}(0,1)}\left|\frac{\partial}{\partial x_{i}} \frac{\partial G}{\partial \boldsymbol{\nu}}(0, \boldsymbol{y})-\frac{\frac{\partial G}{\partial \boldsymbol{\nu}}\left(h \boldsymbol{e}_{i}, \boldsymbol{y}\right)-\frac{\partial G}{\partial \boldsymbol{\nu}}(0, \boldsymbol{y})}{h}\right| \mathrm{d} S(\boldsymbol{y})=0, \\
& \lim _{h \rightarrow 0} \int_{\mathcal{B}(0,1)}\left|\frac{\partial G}{\partial x_{i}}(0, \boldsymbol{y})-\frac{G\left(h \boldsymbol{e}_{i}, \boldsymbol{y}\right)-G(0, \boldsymbol{y})}{h}\right| \mathrm{d} \boldsymbol{y}=0 .
\end{aligned}
$$

Proof. Noting that $\boldsymbol{y} \in \partial \mathcal{B}(0,1)$, we have

$$
\begin{aligned}
\frac{\partial}{\partial x_{i}} \frac{\partial G}{\partial \boldsymbol{\nu}}(0, \boldsymbol{y}) & =\left.\frac{\partial}{\partial x_{i}}\left(\frac{1}{n \alpha(n)} \frac{1-|\boldsymbol{x}|^{2}}{|\boldsymbol{x}-\boldsymbol{y}|^{n}}\right)\right|_{\boldsymbol{x}=0} \\
& =\left.\frac{1}{n \alpha(n)}\left(\frac{2 x_{i}}{|\boldsymbol{x}-\boldsymbol{y}|^{n}}-\frac{n\left(1-|\boldsymbol{x}|^{2}\right)\left(x_{i}-y_{i}\right)}{|\boldsymbol{x}-\boldsymbol{y}|^{n+2}}\right)\right|_{\boldsymbol{x}=0}=\frac{y_{i}}{\alpha(n)} .
\end{aligned}
$$


For the second term of 10 we get

$$
\begin{aligned}
\frac{1}{h}\left(\frac{\partial G}{\partial \boldsymbol{\nu}}\left(h \boldsymbol{e}_{i}, \boldsymbol{y}\right)-\frac{\partial G}{\partial \boldsymbol{\nu}}(0, \boldsymbol{y})\right) & =\frac{1}{n \alpha(n)} \frac{1}{h}\left(\frac{1-h^{2}}{\left|h \boldsymbol{e}_{i}-\boldsymbol{y}\right|^{n}}-1\right) \\
& =\frac{1}{n \alpha(n)} \frac{1}{h}\left(-\frac{h^{2}}{\left|h \boldsymbol{e}_{i}-\boldsymbol{y}\right|^{n}}+\frac{1-\left|h \boldsymbol{e}_{i}-\boldsymbol{y}\right|^{n}}{\left|h \boldsymbol{e}_{i}-\boldsymbol{y}\right|^{n}}\right) .
\end{aligned}
$$

Now using the Taylor expansion

$$
\begin{aligned}
\left|h \boldsymbol{e}_{i}-\boldsymbol{y}\right|^{n} & =\left(\left|h \boldsymbol{e}_{i}-\boldsymbol{y}\right|^{2}\right)^{\frac{n}{2}}=\left(h^{2}-2 h y_{i}+|\boldsymbol{y}|^{2}\right)^{\frac{n}{2}}=\left(1-h\left(2 y_{i}+h\right)\right)^{\frac{n}{2}} \\
& =1-\frac{n}{2} h\left(2 y_{i}+h\right)+\mathcal{O}\left(h^{2}\right)=1-n h y_{i}+\mathcal{O}\left(h^{2}\right),
\end{aligned}
$$

we obtain

$$
\begin{aligned}
\frac{1}{h}\left(\frac{\partial G}{\partial \boldsymbol{\nu}}\left(h \boldsymbol{e}_{i}, \boldsymbol{y}\right)-\frac{\partial G}{\partial \boldsymbol{\nu}}(0, \boldsymbol{y})\right) & =\frac{1}{n \alpha(n)} \frac{1}{h}\left(\frac{1-\left(1-n h y_{i}+\mathcal{O}\left(h^{2}\right)\right)}{\left|h \boldsymbol{e}_{i}-\boldsymbol{y}\right|^{n}}-\frac{h^{2}}{\left|h \boldsymbol{e}_{i}-\boldsymbol{y}\right|^{n}}\right) . \\
& =\frac{1}{n \alpha(n)}\left(n y_{i}+\mathcal{O}(h)\right)=\frac{y_{i}}{\alpha(n)}+\mathcal{O}(h) .
\end{aligned}
$$

Consequently, the function in the first integral of 10 converges uniformly to zero with respect to $\boldsymbol{y} \in \partial \mathcal{B}(0,1)$.

Let us show that the second limit (11) holds true. We start by partitioning the unit ball $\mathcal{B}(0,1)$ as the union of two sets $\mathcal{B}(0, \delta)$ and $\mathcal{B}(0,1)-\mathcal{B}(0, \delta)$, where $0<\delta<1$. Then for the first ball we have

$$
\begin{aligned}
\int_{\mathcal{B}(0, \delta)} & \left|\frac{\partial G}{\partial x_{i}}(0, \boldsymbol{y})-\frac{G\left(h \boldsymbol{e}_{i}, \boldsymbol{y}\right)-G(0, \boldsymbol{y})}{h}\right| \mathrm{d} \boldsymbol{y} \\
& \leqslant \int_{\mathcal{B}(0, \delta)}\left|\frac{\partial G}{\partial x_{i}}(0, \boldsymbol{y})\right| \mathrm{d} \boldsymbol{y}+\frac{1}{h} \int_{\mathcal{B}(0, \delta)}\left|G\left(h \boldsymbol{e}_{i}, \boldsymbol{y}\right)-G(0, \boldsymbol{y})\right| \mathrm{d} \boldsymbol{y} \\
= & I_{1}+I_{2} .
\end{aligned}
$$

Using formula (8) at the point $(0, \boldsymbol{y})$, we compute the integral $I_{1}$ :

$$
I_{1}=\frac{1}{n \alpha(n)} \int_{\mathcal{B}(0, \delta)}\left|\frac{y_{i}}{|\boldsymbol{y}|^{n}}-y_{i}\right| \mathrm{d} \boldsymbol{y}=\frac{1}{n \alpha(n)} \int_{\mathcal{B}(0, \delta)}\left|y_{i}\right|\left|\frac{1-|\boldsymbol{y}|^{n}}{|\boldsymbol{y}|^{n}}\right| \mathrm{d} \boldsymbol{y} \leqslant \frac{1}{n \alpha(n)} \int_{\mathcal{B}(0, \delta)} \frac{1}{|\boldsymbol{y}|^{n-1}} \mathrm{~d} \boldsymbol{y} \text {. }
$$

We apply the definition of $G(\boldsymbol{x}, \boldsymbol{y})$ in $(2)$ at the points $(0, \boldsymbol{y})$ and $\left(h \boldsymbol{e}_{i}, \boldsymbol{y}\right)$ and obtain

$$
I_{2}=\frac{1}{n(n-2) \alpha(n)} \frac{1}{h} \int_{\mathcal{B}(0, \delta)}\left|\left(\frac{1}{\left|h \boldsymbol{e}_{i}-\boldsymbol{y}\right|^{n-2}}-\frac{1}{|\boldsymbol{y}|^{n-2}}\right)+\left(1-\frac{1}{|| \boldsymbol{y} \mid\left(h \boldsymbol{e}_{i}-\left.\frac{\boldsymbol{y}}{|\boldsymbol{y}|^{2}}\right|^{n-2}\right.}\right)\right| \mathrm{d} \boldsymbol{y} .
$$

Now we make use of Lemma A.1. to estimate the expressions inside the brackets. By setting $a=1 /\left|h \boldsymbol{e}_{i}-\boldsymbol{y}\right|$ and $b=\frac{1}{|\boldsymbol{y}|}$ in inequality (25) and by using the fact that $|a|-|b| \leqslant|a-b|$, we can easily get that

$$
\begin{aligned}
\left|\frac{1}{\left|h \boldsymbol{e}_{i}-\boldsymbol{y}\right|^{n-2}}-\frac{1}{|\boldsymbol{y}|^{n-2}}\right| & \leqslant \frac{n-2}{2}|| \boldsymbol{y}|-| h \boldsymbol{e}_{i}-\boldsymbol{y}||\left(\frac{1}{\left|h \boldsymbol{e}_{i}-\boldsymbol{y}\right|^{n-1}}+\frac{1}{|\boldsymbol{y}|^{n-1}}\right) \\
& \leqslant \frac{n-2}{2} h\left(\frac{1}{\left|h \boldsymbol{e}_{i}-\boldsymbol{y}\right|^{n-1}}+\frac{1}{|\boldsymbol{y}|^{n-1}}\right) .
\end{aligned}
$$

In the same way, we let

$$
a=1, \quad b=\frac{1}{|| \boldsymbol{y}\left|h \boldsymbol{e}_{i}-\frac{\boldsymbol{y}}{|\boldsymbol{y}|}\right|},
$$

and by using the fact that

$$
|| \boldsymbol{y}\left|h \boldsymbol{e}_{i}-\frac{\boldsymbol{y}}{|\boldsymbol{y}|}\right| \geqslant\left|\frac{\boldsymbol{y}}{|\boldsymbol{y}|}-\right||\boldsymbol{y}| h \boldsymbol{e}_{i} \mid \geqslant 1-h
$$


we get

$$
\begin{aligned}
\left|1-\frac{1}{|| \boldsymbol{y}\left|\left(h \boldsymbol{e}_{i}-\frac{\boldsymbol{y}}{|\boldsymbol{y}|^{2}}\right)\right|^{n-2}}\right| & \leqslant \frac{n-2}{2}|1-||\boldsymbol{y}| h \boldsymbol{e}_{i}-\frac{\boldsymbol{y}}{|\boldsymbol{y}|}||\left(1+\frac{1}{|| \boldsymbol{y}\left|h \boldsymbol{e}_{i}-\frac{\boldsymbol{y}}{|\boldsymbol{y}|}\right|^{n-1}}\right) \\
& \leqslant \frac{n-2}{2} h\left(1+\frac{1}{(1-h)^{n-1}}\right) .
\end{aligned}
$$

Hence, we obtain the following estimate for the second integral $I_{2}$ :

$$
I_{2} \leqslant \frac{1}{2 n \alpha(n)} \int_{\mathcal{B}(0, \delta)}\left(\frac{1}{\left|h \boldsymbol{e}_{i}-\boldsymbol{y}\right|^{n-1}}+\frac{1}{|\boldsymbol{y}|^{n-1}}\right) \mathrm{d} \boldsymbol{y}+\frac{\delta^{n}}{2 n}\left(1+\frac{1}{(1-h)^{n-1}}\right) .
$$

From the above estimates for $I_{1}$ and $I_{2}$, we observe that the integrals contain two weakly singular integrals at points $\boldsymbol{y}=0$ and $\boldsymbol{y}=h \boldsymbol{e}_{i}$. Letting $h<\delta / 2$, we ensure that both points lie inside the ball $\mathcal{B}(0, \delta)$. Using the standard techniques for computing weakly singular integrals, we get

and

$$
\int_{\mathcal{B}(0, \delta)} \frac{1}{|\boldsymbol{y}|^{n-1}} \mathrm{~d} \boldsymbol{y}=n \alpha(n) \delta
$$

$$
\int_{\mathcal{B}(0, \delta)} \frac{1}{\left|h \boldsymbol{e}_{i}-\boldsymbol{y}\right|^{n-1}} \mathrm{~d} \boldsymbol{y}<\int_{\mathcal{B}\left(h \boldsymbol{e}_{i}, \delta+h\right)} \frac{1}{\left|h \boldsymbol{e}_{i}-\boldsymbol{y}\right|^{n-1}} \mathrm{~d} \boldsymbol{y}=n \alpha(n)(\delta+h) .
$$

Thus, we obtain the following estimate for integral (11)

$$
I_{1}+I_{2}<\delta+\frac{\delta+h}{2}+\frac{\delta}{2}+\frac{\delta^{n}}{2 n}\left(1+\frac{1}{(1-h)^{n-1}}\right) .
$$

In the second set $\mathcal{B}(0,1)-\mathcal{B}(0, \delta)$, the function in the integral $(11)$ is continuous, has no singular points and converges uniformly to zero. Therefore, the integral of this function converges to zero. This completes the proof.

Remark 2. Let $\boldsymbol{x} \in \mathcal{B}^{0}(0,1)$ be a fixed point. Then, following the lines of the proof of Lemma 2.3, one can prove that

$$
\begin{aligned}
& \lim _{h \rightarrow 0} \int_{\partial \mathcal{B} 0,1)}\left|\frac{\partial}{\partial x_{i}} \frac{\partial G}{\partial \boldsymbol{\nu}}(\boldsymbol{x}, \boldsymbol{y})-\frac{\frac{\partial G}{\partial \nu}\left(\boldsymbol{x}+h \boldsymbol{e}_{i}, \boldsymbol{y}\right)-\frac{\partial G}{\partial \nu}(\boldsymbol{x}, \boldsymbol{y})}{h}\right| \mathrm{d} S(\boldsymbol{y})=0, \\
& \lim _{h \rightarrow 0} \int_{\mathcal{B}(0,1)}\left|\frac{\partial G}{\partial x_{i}}(\boldsymbol{x}, \boldsymbol{y})-\frac{G\left(\boldsymbol{x}+h \boldsymbol{e}_{i}, \boldsymbol{y}\right)-G(\boldsymbol{x}, \boldsymbol{y})}{h}\right| \mathrm{d} \boldsymbol{y}=0 .
\end{aligned}
$$

\section{Proof OF THE MAIN THEOREMS}

3.1. Proof of Theorem 2.1. Using the result of Theorem 1.1, we know that $u$ is an almost periodic function. Lemma 2.2 shows that the function $u\left(\boldsymbol{x}_{0}+\boldsymbol{x}\right)$, where $\boldsymbol{x}_{0}$ is a fixed point in $\mathbb{R}^{n}$, is continuously differentiable, and moreover, for its derivatives $\partial u / \partial x_{i}, i=1, \ldots, n$, the following representation

$$
\begin{aligned}
\frac{\partial u}{\partial x_{i}}\left(\boldsymbol{x}_{0}+\boldsymbol{x}\right)= & -\int_{\partial \mathcal{B}(0,1)} u\left(\boldsymbol{x}_{0}+\boldsymbol{y}\right) \frac{\partial}{\partial x_{i}} \frac{\partial G}{\partial \boldsymbol{\nu}}(\boldsymbol{x}, \boldsymbol{y}) \mathrm{d} S(\boldsymbol{y}) \\
& +\int_{\mathcal{B}(0,1)} f\left(\boldsymbol{x}_{0}+\boldsymbol{y}\right) \frac{\partial G}{\partial x_{i}}(\boldsymbol{x}, \boldsymbol{y}) \mathrm{d} \boldsymbol{y} .
\end{aligned}
$$

holds for each $\boldsymbol{x}$ in the unit ball $\mathcal{B}^{0}(0,1)$.

Our aim is to show that the following relation holds:

$$
\lim _{h \rightarrow 0} \sup _{\boldsymbol{x}_{0} \in \mathbb{R}^{n}}\left|\frac{u\left(\boldsymbol{x}_{0}+h \boldsymbol{e}_{i}\right)-u\left(\boldsymbol{x}_{0}\right)}{h}-\frac{\partial u}{\partial x_{i}}\left(\boldsymbol{x}_{0}\right)\right|=0 .
$$


We begin with writing representation formula (4) at the point $\left(\boldsymbol{x}_{0}+\boldsymbol{x}\right)$ for each $\boldsymbol{x} \in \mathcal{B}^{0}(0,1)$ :

$$
u\left(\boldsymbol{x}_{0}+\boldsymbol{x}\right)=-\int_{\partial \mathcal{B}(0,1)} u\left(\boldsymbol{x}_{0}+\boldsymbol{y}\right) \frac{\partial G}{\partial \boldsymbol{\nu}}(\boldsymbol{x}, \boldsymbol{y}) \mathrm{d} S(\boldsymbol{y})+\int_{\mathcal{B}(0,1)} f\left(\boldsymbol{x}_{0}+\boldsymbol{y}\right) G(\boldsymbol{x}, \boldsymbol{y}) \mathrm{d} \boldsymbol{y} .
$$

It follows from relations (17) and $(19)$ that

$$
\begin{aligned}
\left|\frac{u\left(\boldsymbol{x}_{0}+h \boldsymbol{e}_{i}\right)-u\left(\boldsymbol{x}_{0}\right)}{h}-\frac{\partial u}{\partial x_{i}}\left(\boldsymbol{x}_{0}\right)\right| & \\
\leqslant & \sup _{\boldsymbol{x} \in \mathcal{B}(0,1)}|u(\boldsymbol{x})| \int_{\partial \mathcal{B}(0,1)}\left|\frac{1}{h}\left(\frac{\partial G}{\partial \boldsymbol{\nu}}\left(h \boldsymbol{e}_{i}, \boldsymbol{y}\right)-\frac{\partial G}{\partial \boldsymbol{\nu}}(0, \boldsymbol{y})\right)-\frac{\partial}{\partial x_{i}} \frac{\partial G}{\partial \boldsymbol{\nu}}(0, \boldsymbol{y})\right| \mathrm{d} S(\boldsymbol{y}) \\
& +\sup _{\boldsymbol{x} \in \mathcal{B}(0,1)}|f(\boldsymbol{x})| \int_{\mathcal{B}(0,1)}\left|\frac{1}{h}\left(G\left(h \boldsymbol{e}_{i}, \boldsymbol{y}\right)-G(0, \boldsymbol{y})\right)-\frac{\partial G}{\partial x_{i}}(0, \boldsymbol{y})\right| \mathrm{d} \boldsymbol{y} .
\end{aligned}
$$

This inequality and Lemma 2.3 imply relation (18).

According to Theorem 1.1, the function $u(\boldsymbol{x})$ is almost periodic and therefore, $\left(u\left(\boldsymbol{x}_{0}+h \boldsymbol{e}_{i}\right)-\right.$ $\left.u\left(\boldsymbol{x}_{0}\right)\right) / h$, for $h>0, i=1, \ldots, n$, is an almost periodic function. Hence, its limit $\partial u / \partial x_{i}\left(\boldsymbol{x}_{0}\right)$, which is the uniformly continuous limit of this function, is also an almost periodic function. The proof is complete.

3.2. Proof of Theorem 2.2. The proof of the theorem is split into three steps. First, assuming that $u$ is a continuous bounded function, we obtain representation formula (9) for the ball of radius $r$ at the origin $\mathcal{B}(0, r)$. Then, following the lines of the proof of Lemma 2.1. we construct the representation formula for the generalized function $u$ and we prove that $u$ is continuous and bounded in the usual sense at the origin, i.e., $\boldsymbol{x}=0$. Finally, then we prove that $u$ is continuous and bounded for each point in $\mathbb{R}^{n}$.

Step 1. Let us assume for the time being that $u$ is a bounded continuous function. Let us define the Green's function for a ball of radius $r$, i.e., $\mathcal{B}(0, r) \in \mathbb{R}^{n}$ :

$$
G_{r}(\boldsymbol{x}, \boldsymbol{y}):=\Phi(\boldsymbol{y}-\boldsymbol{x})-\Phi\left(\frac{|\boldsymbol{x}|}{r}(\boldsymbol{y}-\tilde{\boldsymbol{x}})\right), \quad((\boldsymbol{x}, \boldsymbol{y}) \in \mathcal{B}(0, r), \boldsymbol{x} \neq \boldsymbol{y}),
$$

where $\tilde{\boldsymbol{x}}=\frac{r^{2} \boldsymbol{x}}{|\boldsymbol{x}|^{2}}$. and $\Phi(\boldsymbol{x})$ is defined as in (3).

Then the representation formula in a ball of radius $r$ can be written as

$$
\begin{aligned}
u(\boldsymbol{x}) & =-\int_{\partial \mathcal{B}(0, r)} u(\boldsymbol{y}) \frac{\partial G_{r}}{\partial \boldsymbol{\nu}}(\boldsymbol{x}, \boldsymbol{y}) \mathrm{d} S(\boldsymbol{y})+\int_{\mathcal{B}(0, r)} f(\boldsymbol{y}) G_{r}(\boldsymbol{x}, \boldsymbol{y}) \mathrm{d} \boldsymbol{y} \\
& =\frac{r^{2}-|\boldsymbol{x}|^{2}}{n \alpha(n) r} \int_{\partial \mathcal{B}(0, r)} \frac{u(\boldsymbol{y})}{|\boldsymbol{x}-\boldsymbol{y}|^{n}} \mathrm{~d} S(\boldsymbol{y})+\int_{\mathcal{B}(0, r)} f(\boldsymbol{y}) G_{r}(\boldsymbol{x}, \boldsymbol{y}) \mathrm{d} \boldsymbol{y} .
\end{aligned}
$$

We multiply $(20)$ by $\varphi(r) r^{n-1} \neq 0$, let $\boldsymbol{x}=0$ and integrate in the variable $r \in[0, R]$ for some $R>r$. By noting that $|\boldsymbol{y}|=r$ we obtain:

$$
\begin{aligned}
u(0) \underbrace{\int_{0}^{R} \varphi(r) r^{n-1} \mathrm{~d} r}_{I_{1}}= & \underbrace{\int_{0}^{R} \frac{\varphi(r) r^{n}}{n \alpha(n) r^{n}} \int_{\partial \mathcal{B}(0, r)} u(\boldsymbol{y}) \mathrm{d} S(\boldsymbol{y}) \mathrm{d} r}_{I_{2}} \\
& +\underbrace{\int_{0}^{R} \varphi(r) r^{n-1} \int_{\mathcal{B}(0, r)} f(\boldsymbol{y}) G_{r}(0, \boldsymbol{y}) \mathrm{d} \boldsymbol{y} \mathrm{d} r}_{I_{3}(f)} .
\end{aligned}
$$

We pass to the spherical coordinates $\left(r, \phi_{1}, \ldots, \phi_{n-1}\right)$, where $r$ is the radial distance, $\phi_{i}$, $i=1, \ldots, n-1$ are angular coordinates, and in view of the identities

$$
\mathrm{d} S(\boldsymbol{y})=\sin ^{n-2}\left(\phi_{1}\right) \sin ^{n-3}\left(\phi_{2}\right) \cdots \sin \left(\phi_{n-2}\right) \mathrm{d} \phi_{1} \mathrm{~d} \phi_{2} \cdots \mathrm{d} \phi_{n-2}, \quad \mathrm{~d} \boldsymbol{y}=r^{n-1} \mathrm{~d} S(\boldsymbol{y}) \mathrm{d} r
$$


we obtain that

$$
I_{2}=\frac{1}{n \alpha(n)} \int_{|y| \leqslant R} \frac{\varphi(|\boldsymbol{y}|) u(\boldsymbol{y})}{|\boldsymbol{y}|^{n-1}} \mathrm{~d} \boldsymbol{y} .
$$

By dividing both parts of (21) by $I_{1}$ we obtain:

$$
u(0)=\frac{1}{n \alpha(n)} \frac{1}{I_{1}} \int_{|y| \leqslant R} \frac{\varphi(|\boldsymbol{y}|) u(\boldsymbol{y})}{|\boldsymbol{y}|^{n-1}} \mathrm{~d} \boldsymbol{y}+\frac{I_{3}(f)}{I_{1}} .
$$

Step 2. Assume that $u$ is a generalized function. By repeating the above steps with $u_{\varepsilon}=u * \omega_{\varepsilon}$ and a corresponding source function $f_{\varepsilon}=f * \omega_{\varepsilon}$, and using in addition Lemma 2.1 the following relation can be obtained:

$$
u_{\varepsilon}(0)=\frac{1}{n \alpha(n)} \frac{1}{I_{1}} \int_{|y| \leqslant R} \frac{\varphi(|\boldsymbol{y}|)}{|\boldsymbol{y}|^{n-1}} u_{\varepsilon}(\boldsymbol{y}) \mathrm{d} \boldsymbol{y}+\frac{1}{I_{1}} I_{3}\left(f_{\varepsilon}\right) .
$$

In our construction, the function $\varphi$ is chosen such that $\varphi(|\boldsymbol{y}|)=0$ as $|\boldsymbol{y}|<\delta$ or $|\boldsymbol{y}|>R-\delta$, where $\delta>0$ is some small number. Then, it is clear that $\psi(\boldsymbol{y}):=\varphi(|\boldsymbol{y}|) /|\boldsymbol{y}|^{n-1}$ is a test function, i.e., it is an element of $\mathcal{D}\left(\mathbb{R}^{n}\right)$. We denote:

$$
v(\boldsymbol{x}):=(u * \psi)(\boldsymbol{x})=(u(\boldsymbol{y}-\boldsymbol{x}), \psi(\boldsymbol{y})) .
$$

Now using Lemma $A \sqrt{.2}$ the integral in the right hand side of $(22)$ can be simplified as follows:

$$
\begin{aligned}
\int_{|\boldsymbol{y}| \leqslant R} \psi(\boldsymbol{y}) u_{\varepsilon}(\boldsymbol{y}) \mathrm{d} \boldsymbol{y} & =\int_{|\boldsymbol{y}| \leqslant R} \psi(\boldsymbol{y})\left(u * \omega_{\varepsilon}\right)(\boldsymbol{y}) \mathrm{d} \boldsymbol{y} \\
& =\int_{\mathbb{R}^{n}} \omega_{\varepsilon}(\boldsymbol{y})(u * \psi)(\boldsymbol{y}) \mathrm{d} \boldsymbol{y} \\
& =\int_{\mathbb{R}^{n}} \omega_{\varepsilon}(\boldsymbol{y}) v(\boldsymbol{y}) \mathrm{d} \boldsymbol{y}=v_{\varepsilon}(0),
\end{aligned}
$$

where $v_{\varepsilon}=v * \omega_{\varepsilon}$. Using identity (23), we rewrite equation (22) in the following form:

$$
u_{\varepsilon}(0)=\frac{1}{n \alpha(n)} \frac{1}{I_{1}} v_{\varepsilon}(0)+\frac{1}{I_{1}} I_{3}\left(f_{\varepsilon}\right) .
$$

This yields that the expression in the right hand side has a limit as $\varepsilon \rightarrow 0$, since $v_{\varepsilon}(0)$ and $f_{\varepsilon}$ have a limit as $\varepsilon \rightarrow 0$. Consequently, we conclude that there exists a limit $u_{\varepsilon}(0)$ as $\varepsilon \rightarrow 0$.

Step 3. In the second part of the proof, under the assumptions of the theorem, we showed that $u$ is continuous and bounded at point $\boldsymbol{x}=0$. Here we show that this is in fact true for each point of the space.

For a fixed point $\boldsymbol{x}_{0} \in \mathbb{R}^{n}$ let us denote $w(\boldsymbol{x}):=u\left(\boldsymbol{x}+\boldsymbol{x}_{0}\right)$, where $w(\boldsymbol{x})$ is a the solution of equation

$$
-\Delta w(\boldsymbol{x})=f_{\boldsymbol{x}_{0}}(\boldsymbol{x}), \quad \boldsymbol{x} \in \mathbb{R}^{n},
$$

in the distribution sense, where we denote $f_{\boldsymbol{x}_{0}}(\boldsymbol{x}):=f\left(\boldsymbol{x}+\boldsymbol{x}_{0}\right)$. Then, we have that $w_{\varepsilon}(\boldsymbol{x})=u_{\varepsilon}\left(\boldsymbol{x}+\boldsymbol{x}_{0}\right)$ and

$$
v\left(\boldsymbol{x}-\boldsymbol{x}_{0}\right)=(u * \psi)\left(\boldsymbol{x}-\boldsymbol{x}_{0}\right)=(w(\boldsymbol{y}-\boldsymbol{x}), \psi(\boldsymbol{y}))=(w * \psi)(\boldsymbol{x}),
$$

and the equation (24) for $w$ becomes:

$$
w_{\varepsilon}(0)=\frac{1}{n \alpha(n)} \frac{1}{I_{1}} v_{\varepsilon}\left(-\boldsymbol{x}_{0}\right)+\frac{1}{I_{1}} I_{3}\left(f_{\boldsymbol{x}_{0}, \varepsilon}\right),
$$

which is

$$
u_{\varepsilon}\left(\boldsymbol{x}_{0}\right)=\frac{1}{n \alpha(n)} \frac{1}{I_{1}} v_{\varepsilon}\left(-\boldsymbol{x}_{0}\right)+\frac{1}{I_{1}} I_{3}\left(f_{\boldsymbol{x}_{0}, \varepsilon}\right),
$$

Now, passing to again the limit as $\varepsilon \rightarrow 0$, we see that $v_{\varepsilon}\left(-\boldsymbol{x}_{0}\right) \rightarrow v\left(-\boldsymbol{x}_{0}\right), f_{\boldsymbol{x}_{0}, \varepsilon} \rightarrow f_{\boldsymbol{x}_{0}}$, and therefore, the limit of $u_{\varepsilon}\left(\boldsymbol{x}_{0}\right)$ exists as $\varepsilon \rightarrow 0$ for each $\boldsymbol{x}_{0} \in \mathbb{R}^{n}$. The proof is complete. 


\section{APPENDIX}

Lemma A.1. Let $a$ and $b$ be arbitrary positive numbers. For each $m=1,2,3, \ldots$ the following inequality holds

$$
\left|a^{m}-b^{m}\right| \leqslant \frac{m}{2}\left|\frac{1}{b}-\frac{1}{a}\right|\left(a^{m+1}+b^{m+1}\right) .
$$

Proof. Using the polynomial identities, we have

$$
a^{m}-b^{m}=(a-b) \sum_{k=1}^{m-1} a^{m-k} b^{k-1}=\frac{a-b}{a b} a b \sum_{k=1}^{m-1} a^{m-k} b^{k-1}=\left(\frac{1}{b}-\frac{1}{a}\right) \sum_{k=0}^{m-1} a^{m-k} b^{k+1} .
$$

Next, we need to use the following Hölder's inequality

$$
d c \leqslant \frac{d^{p}}{p}+\frac{c^{q}}{q}
$$

where

$$
d>0, \quad c>0, \quad p>1, \quad p>1 \quad, \frac{1}{p}+\frac{1}{q}=1 .
$$

By letting

$$
d=a^{m-k}, \quad c=b^{k+1}, \quad p=\frac{m+1}{m-k}, \quad q=\frac{m+1}{k+1}
$$

in the Hölder's inequality, we get

$$
a^{m-k} b^{k+1} \leqslant \frac{m-k}{m+1} a^{m+1}+\frac{k+1}{m+1} b^{m+1} .
$$

Now, we apply this inequality to (26) and obtain

$$
\begin{aligned}
\left|a^{m}-b^{m}\right| & \leqslant\left|\frac{1}{b}-\frac{1}{a}\right|\left(a^{m+1} \sum_{k=0}^{m-1} \frac{m-k}{m+1}+b^{m+1} \sum_{k=0}^{m-1} \frac{k+1}{m+1}\right) \\
& =\frac{m}{2}\left|\frac{1}{b}-\frac{1}{a}\right|\left(a^{m+1}+b^{m+1}\right),
\end{aligned}
$$

which completes the proof of the lemma.

Lemma A.2. Let $u$ be a distribution in $\mathbb{R}^{n}$, and let $\varphi(\boldsymbol{x})$ and $\psi(\boldsymbol{x})$ be two test functions from $\mathcal{D}\left(\mathbb{R}^{n}\right)$ such that $\varphi(-\boldsymbol{x})=\varphi(\boldsymbol{x})$, and $\psi(-\boldsymbol{x})=\psi(\boldsymbol{x})$. Then the following identity holds true:

$$
\int_{\mathbb{R}^{n}} \varphi(\boldsymbol{x})(u * \psi)(\boldsymbol{x}) \mathrm{d} \boldsymbol{x}=\int_{\mathbb{R}^{n}} \psi(\boldsymbol{x})(u * \varphi)(\boldsymbol{x}) \mathrm{d} \boldsymbol{x}
$$

Proof. Let us denote the support of functions $\psi$ and $\varphi$ by $V \subset \mathbb{R}^{n}$. Let $V_{k}, k=1, \ldots, N$, where $N$ is some finite number, be a set of disjoint simplexes such that $\bar{V}=\cup_{k=1, \ldots, N} \bar{V}_{k}$, where $\bar{V}$ and $\bar{V}_{k}$ denote the closure of $V$ and $V_{k}$, respectively. Then for a fixed point $\boldsymbol{x}_{k} \in V_{k}$, we write the following identity:

$$
\begin{aligned}
\int_{\mathbb{R}^{n}} \varphi(\boldsymbol{x})(u * \psi)(\boldsymbol{x}) \mathrm{d} \boldsymbol{x}= & \sum_{k=1}^{N} \int_{V_{k}}\left(\varphi(\boldsymbol{x})(u * \psi)(\boldsymbol{x})-\varphi\left(\boldsymbol{x}_{k}\right)(u * \psi)\left(\boldsymbol{x}_{k}\right)\right) \mathrm{d} \boldsymbol{x} \\
& +\sum_{k=1}^{N} \int_{V_{k}} \varphi\left(\boldsymbol{x}_{k}\right)(u * \psi)\left(\boldsymbol{x}_{k}\right) \mathrm{d} \boldsymbol{x} .
\end{aligned}
$$


Since the integrand in the first integral of the right hand side is continuous, the first integral goes to zero as $k \rightarrow \infty$. On the other hand, since the integrand of the second integral is constant, we use the definition of a distribution to obtain:

$$
\sum_{k=1}^{N} \int_{V_{k}} \varphi\left(\boldsymbol{x}_{k}\right)(u * \psi)\left(\boldsymbol{x}_{k}\right) \mathrm{d} \boldsymbol{x}=\left(u(\boldsymbol{y}), \sum_{k=1}^{N} \varphi\left(\boldsymbol{x}_{k}\right) \psi\left(\boldsymbol{x}_{k}-\boldsymbol{y}\right)\left|V_{k}\right|\right),
$$

where $\left|V_{k}\right|$ denotes the volume $V_{k}$.

We pass to the limit as $k \rightarrow \infty$ and get that

$$
\int_{\mathbb{R}^{n}} \varphi(\boldsymbol{x})(u * \psi)(\boldsymbol{x}) \mathrm{d} \boldsymbol{x}=\left(u(\boldsymbol{y}), \int_{\mathbb{R}^{n}} \varphi(\boldsymbol{x}) \psi(\boldsymbol{x}-\boldsymbol{y}) \mathrm{d} \boldsymbol{x}\right) .
$$

In the same way, for the right hand side of 27 we get

$$
\int_{\mathbb{R}^{n}} \psi(\boldsymbol{x})(u * \varphi)(\boldsymbol{x}) \mathrm{d} \boldsymbol{x}=\left(u(\boldsymbol{y}), \int_{\mathbb{R}^{n}} \psi(\boldsymbol{x}) \varphi(\boldsymbol{x}-\boldsymbol{y}) \mathrm{d} \boldsymbol{x}\right) .
$$

Finally, by using the assumption of the lemma, i.e., $\varphi(-\boldsymbol{x})=\varphi(\boldsymbol{x})$ and $\psi(-\boldsymbol{x})=\psi(\boldsymbol{x})$, we see that

$$
\begin{aligned}
\int_{\mathbb{R}^{n}} \varphi(\boldsymbol{x}) \psi(\boldsymbol{x}-\boldsymbol{y}) \mathrm{d} \boldsymbol{x} & =\int_{\mathbb{R}^{n}} \varphi(\boldsymbol{x}) \psi(\boldsymbol{y}-\boldsymbol{x}) \mathrm{d} \boldsymbol{x} \\
& =\int_{\mathbb{R}^{n}} \psi(\boldsymbol{x}) \varphi(\boldsymbol{y}-\boldsymbol{x}) \mathrm{d} \boldsymbol{x}=\int_{\mathbb{R}^{n}} \psi(\boldsymbol{x}) \varphi(\boldsymbol{x}-\boldsymbol{y}) \mathrm{d} \boldsymbol{x},
\end{aligned}
$$

where we used the property of mollifier. This completes the proof.

\section{REFERENCES}

1. H. Bohr and O. Neugebauer. Über lineare differentialgleichungen mit konstanten koeffizienten und fastperiodischer rechter seite // Nachr. Ges. Wiss. Göttingen, Math.-Phys. 1926, 8-22 (1926).

2. L. C. Evans. Partial differential equations. Amer. Math. Soc., Providence, RI (2010).

3. J. Favard. Sur les équations différentielles linéaires à coefficients presque-périodiques // Acta Math. 51:1, 31-81 (1928).

4. J. Favard. Leçons sur les fonctions presque-périodiques. Gauthier-Villars, Paris (1933).

5. Ė. Muhamadiev. The invertibility of partial differential operators of elliptic type // Dokl. Akad. Nauk SSSR. 205:6, 1292-1295 (1972).

6. G. R. Sell. Almost periodic solutions of linear partial differential equations // J. Math. Anal. Appl. 42:2, 302-312 (1973).

7. Y. Sibuya. Almost periodic solutions of Poisson's equation // Proc. Amer. Math. Soc. 28:1, 195-198 (1971).

8. M. A. Šubin. Almost periodic functions and partial differential operators. // Uspehi Mat. Nauk, 33:2(200),3-47, 247, (1978). [Russian Math. Surveys 33:2, 1-52, (1978)]

Èrgash Muhamadiev

Vologda State University

Lenin str. 15,

160000, Vologda, Russia

E-mail: emuhamadiev@rambler.ru

Murtazo Nazarov

Uppsala University

P.O. Box 256, SE-751 05 Uppsala, Sweden

E-mail: murtazo.nazarov@it.uu.se 